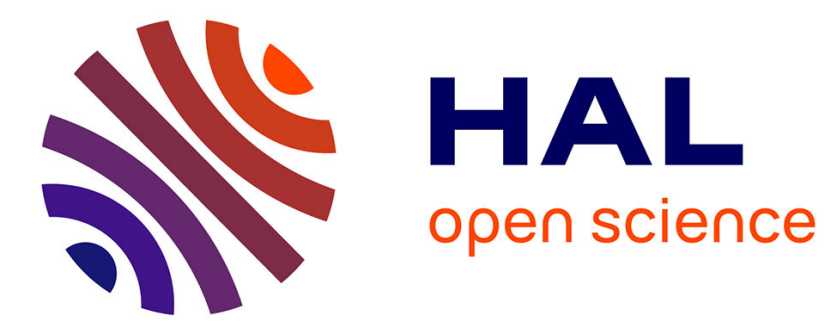

\title{
Acyclic and k-distance coloring of the grid
}

\author{
G. Fertin, Emmanuel Godard, André Raspaud
}

\section{To cite this version:}

G. Fertin, Emmanuel Godard, André Raspaud. Acyclic and k-distance coloring of the grid. Information Processing Letters, 2003, 87 (1), pp.51-58. hal-00307787

\section{HAL Id: hal-00307787 \\ https://hal.science/hal-00307787}

Submitted on 15 Sep 2009

HAL is a multi-disciplinary open access archive for the deposit and dissemination of scientific research documents, whether they are published or not. The documents may come from teaching and research institutions in France or abroad, or from public or private research centers.
L'archive ouverte pluridisciplinaire HAL, est destinée au dépôt et à la diffusion de documents scientifiques de niveau recherche, publiés ou non, émanant des établissements d'enseignement et de recherche français ou étrangers, des laboratoires publics ou privés. 


\title{
Acyclic and $k$-distance Coloring of the Grid
}

\author{
Guillaume Fertin ${ }^{1}$, Emmanuel Godard $^{2}$, André Raspaud $^{2}$ \\ ${ }^{1}$ IRIN UPRES-EA 2157, Université de Nantes \\ 2 rue de la Houssinière - BP 92208 - F44322 Nantes Cedex 3 \\ ${ }^{2}$ LaBRI U.M.R. 5800, Université Bordeaux 1 \\ 351 Cours de la Libération - F33405 Talence Cedex \\ fertin@irin.univ-nantes.fr, \{godard,raspaud\}@labri.u-bordeaux.fr
}

\begin{abstract}
In this paper, we give a relatively simple though very efficient way to color the $d$-dimensional grid $G\left(n_{1}, n_{2} \ldots n_{d}\right)$ (with $n_{i}$ vertices in each dimension $1 \leq i \leq d$ ), for two different types of vertex colorings : (1) acyclic coloring of graphs, in which we color the vertices such that (i) no two neighbors are assigned the same color and (ii) for any two colors $i$ and $j$, the subgraph induced by the vertices colored $i$ or $j$ is acyclic ; and (2) $k$-distance coloring of graphs, in which every vertex must be colored in such a way that two vertices lying at distance less than or equal to $k$ must be assigned different colors. The minimum number of colors needed to acyclically color (resp. $k$-distance color) a graph $G$ is called acyclic chromatic number of $G$ (resp. $k$-distance chromatic number), and denoted $a(G)\left(\right.$ resp. $\left.\chi_{k}(G)\right)$.

The method we propose for coloring the $d$-dimensional grid in those two variants relies on the representation of the vertices of $G_{d}\left(n_{1}, \ldots, n_{d}\right)$ thanks to its coordinates in each dimension ; this gives us upper bounds on $a\left(G_{d}\left(n_{1}, \ldots, n_{d}\right)\right)$ and $\chi_{k}\left(G_{d}\left(n_{1}, \ldots, n_{d}\right)\right)$.

We also give lower bounds on on $a\left(G_{d}\left(n_{1}, \ldots, n_{d}\right)\right)$ and $\chi_{k}\left(G_{d}\left(n_{1}, \ldots, n_{d}\right)\right)$. In particular, we give a lower bound on $a(G)$ for any graph $G$; surprisingly, as far as we know this result was never mentioned before. Applied to the $d$-dimensional grid $G_{d}\left(n_{1}, \ldots, n_{d}\right)$, the lower and upper bounds for $a\left(G_{d}\left(n_{1}, \ldots, n_{d}\right)\right)$ match (and thus give an optimal result) when the lengths in each dimension is "sufficiently large" (more precisely, if $\sum_{i=1}^{d} \frac{1}{n_{i}} \leq 1$ ). If this is not the case, then these bounds differ by an additive constant at most equal to $1-\left\lfloor\sum_{i=1}^{d} \frac{1}{n_{i}}\right\rfloor$. Concerning $\chi_{k}\left(G_{d}\left(n_{1}, \ldots, n_{d}\right)\right)$, we give exact results on its value for (1) $k=2$ and any $d \geq 1$, and (2) $d=2$ and any $k \geq 1$.

In the case of acyclic coloring, we also apply our results to hypercubes of dimension $d$, $H_{d}$, which are a particular case of $G_{d}\left(n_{1}, \ldots, n_{d}\right)$ in which there are only 2 vertices in each dimension. In that case, the bounds we obtain differ by a multiplicative constant equal to 2 .
\end{abstract}

Keywords: acyclic coloring, $k$-distance coloring, $d$-dimensional grids, hypercubes.

In this paper, we consider two types of coloring : acyclic coloring and $k$-distance coloring. Those two colorings are vertex colorings, and are formally defined in Definitions 1 and 2 below.

Definition 1 (Acyclic Coloring) Let $G=(V, E)$ be a graph. An acyclic coloring of $G$ is a vertex coloring of $G$, satisfying the two following rules :

(a) No two neighboring vertices are assigned the same color (this is also denoted as proper coloring).

(b) Let $V_{a} \subseteq V$ (resp. $V_{b} \subseteq V$ ) be the set of vertices of $G$ that are assigned color a (resp. color b). Then, for any $a \neq b$, the subgraph $G^{\prime}$ of $G$ induced by $V_{a} \cup V_{b}$ must be acyclic. 
In other words, an acyclic coloring of $G$ is a vertex coloring of $G$ that is proper, and such that any two classes of colors induce a graph $G^{\prime}$ which is a forest (that is, an acyclic graph).

Definition 2 ( $k$-distance Coloring) Let $G=(V, E)$ be a graph. $A k$-distance coloring of $G$ is a vertex coloring of $G$ satisfying the following rule : no two vertices lying at distance less than or equal to $k$ in $G$ are assigned the same color.

We note that proper coloring is a particular case of $k$-distance coloring, where $k=1$.

The minimum number of colors necessary to acyclically color $G$ is called acyclic chromatic number of $G$, and is denoted $a(G)$. Similarly, the $k$-distance chromatic number of a graph $G$ is the minimum number of colors necessary to $k$-distance color $G$, and is denoted by $\chi_{k}(G)$. We also note that $\chi_{1}(G) \leq a(G) \leq \chi_{2}(G)$ for any graph $G$.

For a family $\mathcal{F}$ of graphs, the acyclic chromatic number of $\mathcal{F}$, denoted by $a(\mathcal{F})$, is defined as the maximum $a(G)$ over all graphs $G \in \mathcal{F}$; and a similar definition holds for the $k$-distance chromatic number of families $\mathcal{F}$ of graphs.

Acyclic coloring has been largely studied in the past 25 years ; in particular, several authors have determined $a(\mathcal{F})$ for several families $\mathcal{F}$ of graphs such as planar graphs [Bor79], planar graphs with "large" girth [BKW99], outerplanar graphs (see for instance [Sop97]), graphs of maximum degree 3 [Grü73], of maximum degree 4 [Bur79], etc. Whereas $k$-distance coloring has been mostly studied in the case where $k=2$; results on the $k$-distance coloring of the hypercube $H_{d}$ are given in [KDP00], while some general asymptotic results about the order of $\chi_{k}(G)$ for any graph $G$, in function of its maximum degree and its girth, are given in [AM00].

In this paper, we consider $d$-dimensional grids $G_{d}\left(n_{1}, \ldots, n_{d}\right)$, where each $n_{i} \geq 2$. Our aim here is to describe a somewhat general method to acyclically and $k$-distance color $G_{d}\left(n_{1}, \ldots, n_{d}\right)$, and thus, give an upper bound on the chromatic number for each of those two colorings. The method varies in the details depending of the considered coloring, but remains the same in spirit in both cases. It relies on the representation of each vertex of $G_{d}\left(n_{1}, \ldots, n_{d}\right)$ by a vector of its coordinates in each dimension.

We also give lower bounds on the acyclic and $k$-distance chromatic number of $G_{d}\left(n_{1}, \ldots, n_{d}\right)$, that match with the upper bound given by our method in most cases ; when the bounds do not match, they differ by an additive factor depending on the value of each $n_{i}, 1 \leq i \leq d$.

Our paper is organized as follows : in Section 1, we first give a lower bound for $a(G)$ that applies for any graph $G$; this bound is then be simplified in order to be used in general $d$-dimensional grids $G_{d}\left(n_{1}, \ldots, n_{d}\right)$. We then give an upper bound on $a\left(G_{d}\left(n_{1}, \ldots, n_{d}\right)\right)$, which we prove to be optimal when the $G_{d}\left(n_{1}, \ldots, n_{d}\right)$ is "sufficiently large". We then turn to hypercubes of dimension $d, H_{d}$ for which we determine $a\left(H_{d}\right)$ for any $d$ up to a multiplicative factor equal to 2 . In Section 2 , we turn to the case of $k$-distance coloring. Here, we consider two subcases : (1) $k=2$ and general $d \geq 1$, for which we give the exact result of $\chi_{2}\left(G_{d}\left(n_{1}, \ldots, n_{d}\right)\right)$; and (2) $d=2$ and general $k \geq 1$, for which we give the exact value of $\chi_{k}\left(G\left(n_{1}, n_{2}\right)\right)$.

\section{Acyclic Coloring}

Before turning to $d$-dimensional grids $G_{d}\left(n_{1}, \ldots, n_{d}\right)$, we first give a lower bound on $a(G)$, for any graph $G$. As far as we know, and to our great surprise, we were not able to find it mentioned anywhere.

Theorem 1 For any graph $G=(V, E)$, let $n=|V|, m=|E|$ and $\Delta=4 n(n-1)-8 m+1$. In that case, we have :

$$
a(G) \geq \frac{2 n+1-\sqrt{\Delta}}{2}
$$

Proof : Let $a(G)=p$. Let $V_{i}, 1 \leq i \leq p$, be the set of vertices whose color is $i$ in an acyclic coloring of $G$ using $p$ colors. By definition, the subgraph of $G$ induced by any $V_{i} \cup V_{j}, 1 \leq i<j \leq p$, 
is a forest. Let $e_{i, j}$ be the set of edges covered by this forest. Clearly, for any two distinct pairs $\left(i_{1}, j_{1}\right)$ and $\left(i_{2}, j_{2}\right)$ with $1 \leq i_{1}<j_{1} \leq p, 1 \leq i_{2}<j_{2} \leq p, e_{i_{1}, j_{1}} \cap e_{i_{2}, j_{2}}=\emptyset$.

It can be easily seen that the number of pairwise distinct pairs of colors is equal to $\frac{p(p-1)}{2}$, and that over those $\frac{p(p-1)}{2}$ pairs of colors, each color $1 \leq k \leq p$ appears $p-1$ times. Moreover, for each pair $(i, j)$, with $1 \leq i<j \leq p$, we have $\left|e_{i, j}\right| \leq\left|V_{i}\right|+\left|V_{j}\right|-1$. It also clearly holds that $\sum_{(i, j)}\left|e_{i, j}\right|=m$.

Combining those two results, we then get : $m \leq \sum_{(i, j)}\left|V_{i}\right|+\left|V_{j}\right|-1$, that is $m \leq(p-$ 1) $\left(\sum_{k=1}^{p}\left|V_{k}\right|\right)-\frac{p(p-1)}{2}$. Since $\sum_{k=1}^{p}\left|V_{k}\right|=n$, we then get : $m \leq n(p-1)-\frac{p(p-1)}{2}$, which gives $p^{2}-(2 n+1) p+2(m+n) \leq 0$. Let $\Delta=4 n(n-1)-8 m+1 . \Delta \geq 1$ in all the cases, since we always have $m \leq \frac{n(n-1)}{2}$. Thus we conclude that $\frac{2 n+1-\sqrt{\Delta}}{2} \leq p \leq \frac{2 n+1+\sqrt{\Delta}}{2}$. However, we can see that the upper bound is not relevant, since we always have $m \leq \frac{n(n-1)}{2}$, that is $\Delta \geq 1$; hence the least value for $\frac{2 n+1+\sqrt{\Delta}}{2}$ is $n+1$. However, it is obvious that $p \leq n$ in all the cases.

It is not difficult to see that this general lower bound is optimal for several different families of graphs, such as trees (in that case, $\Delta=(2 n-3)^{2}$, and thus $a(G) \geq 2$ ), cycles ((in that case, $2 n-4<\sqrt{\Delta}<2 n-3$, and thus $a(G) \geq 3$ ), and complete graphs (in that case, $\Delta=1$, and thus $a(G) \geq n)$.

We will see in the following that it is also optimal in sufficiently large $d$-dimensional grids $G_{d}\left(n_{1}, \ldots, n_{d}\right)$. For this, we will use a slight approximation of the above expression ; though it then loses precision, it will simplify our computations in the rest of the paper.

Proposition 1 Let $G$ be a graph with $n>0$ vertices and $m>0$ edges. Then $a(G) \geq 2+\left\lfloor\frac{m}{n}\right\rfloor$.

Proof : By Theorem 1, we know that for any graph $G=(V, E)$ with $|V|=n$ and $|E|=m$, we have $a(G) \geq \frac{2 n+1-\sqrt{\Delta}}{2}$, with $\Delta=4 n(n-1)-8 m+1$. This gives the following inequality : $a(G) \geq \frac{(2 n+1)^{2}-\Delta}{2(2 n+1+\sqrt{\Delta})}$, that is $a(G) \geq \frac{4(m+n)}{1+2 n+\sqrt{\Delta}}$. This can also be written as follows :

$$
a(G) \geq \frac{m+n}{n} \cdot \frac{4 n}{1+2 n+\sqrt{\Delta}}
$$

Because $\Delta=(2 n-1)^{2}-8 m$, we have $\Delta<(2 n-1)^{2}$, that is $1+2 n+\sqrt{\Delta}<4 n$. Hence $a(G)>1+\frac{m}{n}$.

For instance, we can note that this bound is not tight anymore for complete graphs. However, this approximation will be good enough for our purpose.

We now turn to the specific case of $d$-dimensional grids $G_{d}\left(n_{1}, \ldots, n_{d}\right)$. We first recall formally the definition of $G_{d}\left(n_{1}, \ldots, n_{d}\right)$.

Definition 3 Let $d \in \mathbb{N}$ and $\left(n_{1}, \ldots, n_{d}\right) \in \mathbb{N}^{d}$, with $n_{i} \geq 2$ for any $1 \leq i \leq d$. The $d$-dimensional grid of lengths $n_{1}, \ldots, n_{d}$, denoted by $G_{d}\left(n_{1}, \ldots, n_{d}\right)$, is the following graph:

$$
\begin{gathered}
V\left(G_{d}\left(n_{1}, \ldots, n_{d}\right)\right)=\left[1, n_{1}\right] \times\left[1, n_{2}\right] \times \cdots \times\left[1, n_{d}\right] \\
E\left(G_{d}\left(n_{1}, \ldots, n_{d}\right)\right)= \\
\left\{\{u, v\} \mid u=\left(u_{1}, \ldots, u_{d}\right), v=\left(v_{1}, \ldots, v_{d}\right), \text { and there exist } i_{0}\right. \text { such that } \\
\left.\forall i \neq i_{0}, u_{i}=v_{i}, \text { and }\left|u_{i_{0}}-v_{i_{0}}\right|=1\right\}
\end{gathered}
$$

We recall that for any $d$-dimensional grid $G_{d}\left(n_{1}, \ldots, n_{d}\right)$, we have :

$$
\begin{aligned}
\left|V\left(G_{d}\left(n_{1}, \ldots, n_{d}\right)\right)\right| & =n_{1} \times \cdots \times n_{d} \\
\left|E\left(G_{d}\left(n_{1}, \ldots, n_{d}\right)\right)\right| & =n_{1} \times \cdots \times n_{d} \times\left(d-\sum_{i=1}^{d} \frac{1}{n_{i}}\right)
\end{aligned}
$$

If we now apply Proposition 1 , a lower bound for $G_{d}\left(n_{1}, \ldots, n_{d}\right)$ is then the following. 
Corollary 1 Let $n_{1}, \ldots, n_{d} \in \mathbb{N}$, with $n_{i} \geq 2$ for any $1 \leq i \leq d$. For any grid $G_{d}\left(n_{1}, \ldots, n_{d}\right)$ of dimension $d$,

$$
a\left(G_{d}\left(n_{1}, \ldots, n_{d}\right)\right) \geq 2+\left\lfloor d-\sum_{i=1}^{d} \frac{1}{n_{i}}\right\rfloor
$$

We now give a coloring of any $d$-grid that uses $d+1$ colors.

Theorem 2 Let $n_{1}, \ldots, n_{d} \in \mathbb{N}$ with $n_{i} \geq 2$ for any $1 \leq i \leq d$. For any grid $G_{d}\left(n_{1}, \ldots, n_{d}\right)$ of dimension $d$,

$$
a\left(G_{d}\left(n_{1}, \ldots, n_{d}\right)\right) \leq d+1
$$

Proof : Each vertex $u$ of $G_{d}\left(n_{1}, \ldots, n_{d}\right)$ is defined by its coordinates, ie $u=\left(x_{1}, x_{2} \ldots x_{d}\right)$, where $0 \leq x_{i} \leq n_{i}-1$. Let us define the following coloring : each vertex $u=\left(x_{1}, x_{2} \ldots x_{d}\right)$ is assigned color $c(u)=\left(\sum_{i=1}^{d} i \cdot x_{i}\right) \bmod d+1$. Clearly, this coloring uses no more than $d+1$ colors.

Let us first show that this is a proper coloring. For this, assume that two neighbors $u$ and $u^{\prime}$ are assigned the same color $c$. Assume also that the coordinates of $u$ and $u^{\prime}$ differ on the $j$-th dimension. Since $u=\left(x_{1}, x_{2} \ldots x_{j-1}, x_{j}, x_{j+1} \ldots x_{d}\right)$ and $u^{\prime}=\left(x_{1}, x_{2} \ldots x_{j-1}, x_{j} \pm 1, x_{j+1} \ldots x_{d}\right)$, by definition of $c(u)$ and $c\left(u^{\prime}\right)$ we have $j \cdot x_{j}+\sum_{i=1, i \neq j}^{d} i \cdot x_{i} \equiv(j \pm 1) \cdot x_{j}+\sum_{i=1, i \neq j}^{d} i \cdot x_{i} \bmod$ $d+1$. Thus we end up with $\pm j \equiv 0 \bmod d+1$. However, $j \in[1 ; d]$, hence this is impossible.

Now let us prove that this is an acyclic coloring. Let us take any two distinct colors $c_{1}$ and $c_{2}$ from this coloring, and assume two neighbors $u_{1}$ and $u_{2}$ in $G_{d}\left(n_{1}, \ldots, n_{d}\right)$ are assigned colors $c_{1}$ and $c_{2}$, respectively. Suppose that the coordinates of $u_{1}$ and $u_{2}$ differ on dimension $j$. By definition of the coloring, we end up with the following equality : $c_{1}-c_{2} \equiv \pm j \bmod d+1$. W.l.o.g., suppose that $c_{1}>c_{2}$. Thus there exists only one neighbor $u_{2}^{\prime}$ of $u_{1}$ which is not $u_{2}$, and for which $c\left(u_{2}^{\prime}\right)=c\left(u_{2}\right)=c_{2}$. This neighbor $u_{2}^{\prime}$ differs in the $(d+1-j)$-th coordinate from $u_{2}$. Now, if we are looking for a neighbor of $u_{2}^{\prime}$ which is not $u_{1}$ and which is assigned color $c_{1}$, using the same arguments, we see that it must differ from $u_{1}$ in the $j$-th coordinate. By induction, we see that for any distinct pair of colors $c_{1}$ and $c_{2}$ with $c_{1}>c_{2}$, the vertices which are assigned either $c_{1}$ or $c_{2}$ form a cycle iff they lie in the same 2-dimensional subgrid of $G_{d}\left(n_{1}, \ldots, n_{d}\right)$; this subgrid $G_{2}\left(c_{1}, c_{2}\right)$ being induced by dimension $j=c_{1}-c_{2}$ and $j^{\prime}=d+1-j$ (cf. Figure 1).

No bicolored cycle can exist in that case, because in $G_{2}\left(c_{1}, c_{2}\right)$, the only authorized "moves" between a given pair of colors will be either $\mathrm{N}$ and W, N and E, S and W, or S and E. Hence, the path will be "stair-like", and no cycle can be created.

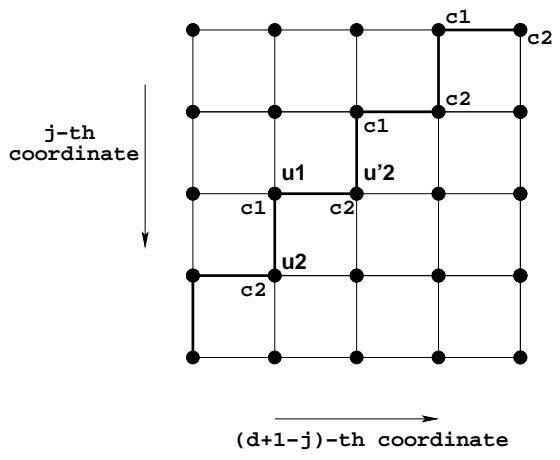

Figure 1: $G_{2}\left(c_{1}, c_{2}\right)$, the subgraph of $G_{d}\left(n_{1}, \ldots, n_{d}\right)$ induced by dimensions $j=c_{1}-c_{2}$ and $j^{\prime}=d+1-j$

Thanks to Theorem 2 above, we are now able to derive tight bounds on $a\left(G_{d}\left(n_{1}, \ldots, n_{d}\right)\right)$, when the grid of dimension $d \geq 2$ is "sufficiently large". 
Theorem 3 Let $d \geq 2$ and $n_{1}, \ldots, n_{d} \in \mathbb{N}$, with $n_{i} \geq 2$ for any $1 \leq i \leq d$. For any grid $G_{d}\left(n_{1}, \ldots, n_{d}\right)$, if $\sum_{i=1}^{\bar{d}} \frac{1}{n_{i}} \leq 1$ then $a\left(G_{d}\left(n_{1}, \ldots, n_{d}\right)\right)=d+1$.

We note that this is true in particular when the minimal length is greater or equal to $d$.

If we now apply the above result to hypercubes of dimension $d, H_{d}$ (which are a particular case of $d$-dimensional grids $G_{d}\left(n_{1}, \ldots, n_{d}\right)$ where each $\left.n_{i}=2,1 \leq i \leq d\right)$, we get the following bounds, that differ by a multiplicative factor 2 .

Theorem 4 For any hypercube $H_{d}$ of dimension $d \geq 1,\left\lceil\frac{d+3}{2}\right\rceil \leq a\left(H_{d}\right) \leq d+1$.

Table 1 shows where the actual values of $a\left(H_{d}\right)$ stand compared to the bounds given in Theorem 4 , for $1 \leq d \leq 6$. The middle column indicates the actual value of $a\left(H_{d}\right)$ (obtained by exhaustive search by computer), while the left (resp. right) column indicates the lower bound (resp. upper bound) from Theorem 4. Bounds that match the actual results are given in bold characters. Though the examples are limited to dimensions going from 1 to 6 , this Table seems to suggest that the lower bound is closer to the actual value than the upper bound.

\begin{tabular}{||c|c|c|c||}
\hline \hline$d$ & $\begin{array}{c}a\left(H_{d}\right) \geq \\
(\text { Theorem } 4)\end{array}$ & $\begin{array}{c}a\left(H_{d}\right)= \\
(\text { Computer })\end{array}$ & $\begin{array}{c}a\left(H_{d}\right) \leq \\
(\text { Theorem } 4)\end{array}$ \\
\hline \hline 1 & $\mathbf{2}$ & 2 & $\mathbf{2}$ \\
\hline 2 & $\mathbf{3}$ & 3 & $\mathbf{3}$ \\
\hline 3 & 3 & 4 & $\mathbf{4}$ \\
\hline 4 & $\mathbf{4}$ & 4 & 5 \\
\hline 5 & $\mathbf{4}$ & 4 & 6 \\
\hline 6 & $\mathbf{5}$ & 5 & 7 \\
\hline \hline
\end{tabular}

Table 1: Where the actual results for $a\left(H_{d}\right)$ stand $(1 \leq d \leq 6)$

Remark 1 The above work on acyclic coloring has also given birth to results concerning a third type of coloring, called star coloring. A star coloring of a graph $G$ is a proper coloring of its vertices, such that no path of length 3 in $G$ is bicolored (in other words, any two classes of colors induce a graph $G^{\prime}$ which is a forest of stars). $\chi_{s}(G)$ denotes the star chromatic number of a graph $G$, that is the minimum number of colors necessary to star color $G$.

Thanks to the above work, in [FRR02] the authors have been able to prove the two following results :

- $2+\left\lfloor d-\sum_{i=1}^{d} \frac{1}{n_{i}}\right\rfloor \leq \chi_{s}\left(G_{d}\left(n_{1}, \ldots, n_{d}\right)\right) \leq 2 d+1$

- $\left\lceil\frac{d+3}{2}\right\rceil \leq \chi_{s}\left(H_{d}\right) \leq d+1$

This shows that the star chromatic number of $G_{d}\left(n_{1}, \ldots, n_{d}\right)$ is determined within a factor never exceeding 4, and that when $G_{d}\left(n_{1}, \ldots, n_{d}\right)$ is "sufficiently large", this factor is reduced to 2. Concerning hypercubes of dimension $d, H_{d}$, the bounds differ by a factor 2.

\section{$2 \quad k$-Distance Coloring}

In this section, we study the $k$-distance coloring of the $d$-dimensional grid. We note that $k$ distance coloring can also be seen as a proper coloring of the $k$-th power of $G, G^{k}$ (we recall that the $k$-th power of a graph $G$ is the graph $G$ to which we add edges between any pair of vertices lying at distance less than or equal to $k$ ).

Here, we first study the particular case $k=2$. We give in this case the exact value of $\chi_{2}\left(G_{d}\left(n_{1}, \ldots, n_{d}\right)\right)$, for any $d \geq 1$. Then, we turn to the case of 2 -dimensional grids, for which we give the exact value of $\chi_{k}\left(G_{2}\right)$, for any $k \geq 1$. 
Theorem $5 \chi_{2}\left(G_{d}\left(n_{1}, \ldots, n_{d}\right)\right)=2 d+1$ for any $d \geq 1$.

Proof: The lower bound comes from the fact that if $n_{i} \geq 3$ for all $1 \leq i \leq d$, there exists in $G_{d}\left(n_{1}, \ldots, n_{d}\right)$ a vertex $u$ for which there are $2 d+1$ vertices lying at distance 2 from $u$ ( $u$ included). Thus, if we denote by $B_{1}(u)$ this set of vertices (the ball of radius 1 around $u$ ), we conclude that any 2-distance coloring will require at least $\left|B_{1}(u)\right|$ colors, since any two vertices $u_{1}$ and $u_{2}$ in $B_{1}(u)$ satisfy $d\left(u_{1}, u_{2}\right) \leq 2$. Thus $\chi_{2}\left(G_{d}\left(n_{1}, \ldots, n_{d}\right)\right) \geq 2 d+1$.

The upper bound is obtained by the following coloring, inspired by the same kind of technique as for the acyclic coloring : if any vertex $u \in V\left(G_{d}\left(n_{1}, \ldots, n_{d}\right)\right)$ is defined thanks to its coordinates (that is, $u=\left(x_{1}, x_{2}, \ldots x_{d}\right)$ with $0 \leq x_{i} \leq n_{i}-1$ for all $\left.1 \leq i \leq d\right)$, then $u$ is assigned color $c(u)=\sum_{i=1}^{d} i x_{i}$ mod $2 d+1$. We now show that this coloring is a 2 -distance coloring of $G_{d}\left(n_{1}, \ldots, n_{d}\right)$. This is done by contradiction : assume that there exist in $G_{d}\left(n_{1}, \ldots, n_{d}\right)$ two distinct vertices $u=\left(x_{1}, x_{2} \ldots x_{d}\right)$ and $v=\left(x_{1}^{\prime}, x_{2}^{\prime} \ldots x_{d}^{\prime}\right)$, such that $d(u, v) \leq 2$, and such that $c(u)=c(v)$. We will show that this cannot happen. For this, we distinguish two cases :

Case $1: d(u, v)=1$. This means that $u$ and $v$ differ on only one coordinate, say $j(1 \leq j \leq d)$. In other words, $x_{j}^{\prime}=x_{j} \pm 1$. Thus, since $c(u)=c(v)$, we have by definition of the coloring $\sum_{i=1}^{d} i x_{i} \equiv \sum_{i=1}^{d} i x_{i}^{\prime} \bmod 2 d+1$. That is, $j x_{j} \equiv j x_{j}^{\prime} \bmod 2 d+1$, or $\pm j \equiv 0 \bmod 2 d+1$. However, $1 \leq j \leq d$, hence this is impossible.

Case $2: d(u, v)=2$. This means that $u$ and $v$ differ on coordinates $j_{1}$ and $j_{2}$ (where we possibly have $j_{1}=j_{2}$ ). In other words, $x_{j_{1}}=x_{j_{1}}^{\prime} \pm 1$ and $x_{j_{2}}=x_{j_{2}}^{\prime} \pm 1$. Since we suppose $c(u)=c(v)$, we have $j_{1} x_{j_{1}}+j_{2} x_{j_{2}} \equiv j_{1} x_{j_{1}}^{\prime}+j_{2} x_{j_{2}}^{\prime} \bmod 2 d+1$, that is $\pm j_{1} \pm j_{2} \equiv 0 \bmod 2 d+1$. Since $1 \leq j_{1}, j_{2} \leq d$, the only possibility is when $j_{1}=j_{2}$; however, when $j_{1}=j_{2}$ and $d(u, v)=2$, then $x_{j_{1}}=x_{j_{1}}^{\prime} \pm 2$, and we end up with $\pm 2 j_{1} \equiv 0 \bmod 2 d+1$, which is impossible since $1 \leq j_{1} \leq d$.

We thus conclude that no two distinct vertices lying at distance less than or equal to 2 can be assigned the same color by the above coloring ; hence, this is a 2-distance coloring and $\chi_{2}\left(G_{d}\left(n_{1}, \ldots, n_{d}\right)\right) \leq 2 d+1$. Altogether, we then have $\chi_{2}\left(G_{d}\left(n_{1}, \ldots, n_{d}\right)\right)=2 d+1$, and the theorem is proved.

Theorem 6 Let $G_{2}$ be the 2-dimensional grid $G\left(n_{1}, n_{2}\right)$, and $k \geq 1$ be an integer. Then, for sufficiently large $n_{1}$ and $n_{2}$ :

- if $k$ is even, then $\chi_{k}\left(G_{2}\right)=\frac{(k+1)^{2}+1}{2}$;

- if $k$ is odd, then $\chi_{k}\left(G_{2}\right)=\frac{(k+1)^{2}}{2}$.

Proof : Suppose first that $k=2 m$ is even. In that case, it can be easily seen that for sufficiently large $n_{1}$ and $n_{2}$ there exists a vertex $v \in V\left(G_{2}\right)$ for which $2 m^{2}+2 m+1$ lie at distance less than or equal to $m$ from $v$ ( $v$ included). Thus, all those vertices, lying in a ball of diameter $k$, must be assigned pairwise distinct colors. We then conclude that $\chi_{k}\left(G_{2}\right) \geq 2 m^{2}+2 m+1$, that is $\chi_{k}\left(G_{2}\right) \geq \frac{(k+1)^{2}+1}{2}$.

The upper bound is given by the following coloring : let any vertex $u \in V\left(G_{2}\right)$ be defined by its coordinates in $G_{2}$. That is, $u=(x, y)$, with $0 \leq x \leq n_{1}-1$ and $0 \leq y \leq n_{2}-1$. Then every vertex $u=(x, y)$ is assigned color $c(u)=x+(k+1) y \bmod p$, where $p=2 m^{2}+2 m+1$. Let us now show by contradiction that this coloring is a $k$-distance coloring for $G_{2}$. For this, let us take two vertices $u$ and $v$ in $V\left(G_{2}\right)$ such that $c(u)=c(v)$, and suppose that $1 \leq d(u, v) \leq k$. Let $u=\left(x_{1}, y_{1}\right)$ and $v=\left(x_{2}, y_{2}\right)=\left(x_{1} \pm s, y_{1} \pm t\right)$, with $s, t \geq 0$ such that $s+t=d(u, v)$. Then by definition of the coloring, $c(u)=c(v)$ is equivalent to $x_{1}+(k+1) y_{1} \equiv x_{1} \pm s+(k+1)\left(y_{1} \pm t\right) \bmod$ $p$. Hence, we have $\pm s \pm(k+1) t \equiv 0 \bmod p$. Let $\kappa=d(u, v)=s+t($ we recall that $1 \leq \kappa \leq k)$. We now distinguish two cases, depending on the signs of the expression :

Case $1: s+(k+1) t \equiv 0 \bmod p$ (also equivalent to $-s-(k+1) t \equiv 0 \bmod p$ ). In other words, $(s+t)+k t \equiv 0 \bmod p$, that is $\kappa+k t=\alpha p$. Now let us discuss the possible values of 
$\alpha$. Clearly, $\alpha$ cannot be equal to zero, since $\kappa \geq 1, k \geq 1$ and $t \geq 0$. If $\alpha \geq 2$, then we have $\kappa+k t \geq 2 p$, where $p=2 m^{2}+2 m+1$, that is $p=\frac{k^{2}}{2}+k+1$. This gives $\kappa+k t \geq k^{2}+2 k+2$, which is impossible since $\kappa \leq k$ and $t \leq k$ (indeed, if $t>k$, then $d(u, v)>k$ ). The only remaining case is $\alpha=1$. This then gives $\kappa+k t=\frac{k^{2}}{2}+k+1$, that is $t=\frac{k}{2}+1+\frac{1-\kappa}{k}$. We recall that $k$ is even, thus the only possibility for $t$ to be an integer is when $b=\frac{1-\kappa}{k}$ is a (possibly negative) integer. However, it is easy to see that this is the case only when $\kappa=1$ (otherwise, $-1<b<0$ ). Hence, we have $\kappa=1$ and $b=0$. But then, $t=\frac{k}{2}+1+b$ becomes $t=\frac{k}{2}+1$. That is, $t>\kappa$, a contradiction.

Case $2: s-(k+1) t \equiv 0 \bmod p($ also equivalent to $-s+(k+1) t \equiv 0 \bmod p)$. In that case, we have $(s+t)-(k+2) t \equiv 0 \bmod p$, that is $t \equiv \frac{\kappa}{k+2}+\alpha p$, where $\alpha$ is an integer. However, it can be easily seen that $p>k$, and that $\frac{\kappa}{k+2}<1$ (since $\kappa \leq k$ ). Thus, since $t$ must be positive and less than or equal to $k$, the only possibility is $\alpha=0$. But in that case, $t=\frac{\kappa}{k+2}$, thus $0<t<1$ cannot be an integer, a contradiction.

Altogether, this proves that any two vertices $u$ and $v$ lying at distance less than or equal to $k$ in $G_{d}\left(n_{1}, \ldots, n_{d}\right)$ cannot be assigned the same color. Thus, our coloring is a $k$-distance coloring. Since this coloring uses $p=\frac{k^{2}}{2}+k+1$ colors, we conclude that $\chi_{k}\left(G_{2}\right) \leq \frac{(k+1)^{2}+1}{2}$.

Altogether, we have that $\chi_{k}\left(G_{2}\right)=\frac{(k+1)^{2}+1}{2}$ for any even $k \geq 2$.

When $k=2 m+1$ is odd, the proof method is similar. The lower bound is given by the following observation : for $n_{1}$ and $n_{2}$ sufficiently large, there exists a vertex $u$ for which $2 m^{2}+2 m+1$ vertices lie at distance less than or equal to $m$ from $u$ ( $u$ included). Let $B_{m}(u)$ be this set of vertices (the ball of radius $m$ around $u$ ) ; it is possible to extend this ball such that it contains $2 m+1$ more vertices, yielding a set $B_{m}^{\prime}(u)$, in such a way that any two vertices $u_{1}$ and $u_{2}$ in $B_{m}^{\prime}(u)$ lie at distance less than or equal to $k=2 m+1$ (cf. Figure 2). This is simply due to the fact that $k$ is odd. Hence, we need to color all the vertices of $B_{m}^{\prime}(u)$ with pairwise distinct colors in order to get a $k$-distance coloring of $G_{2}$. Thus $\chi_{k}\left(G_{2}\right)=\left|B_{m}^{\prime}(u)\right|$, that is $\chi_{k}\left(G_{2}\right)=2 m^{2}+4 m+2$, or $\chi_{k}\left(G_{2}\right) \geq \frac{(k+1)^{2}}{2}$.

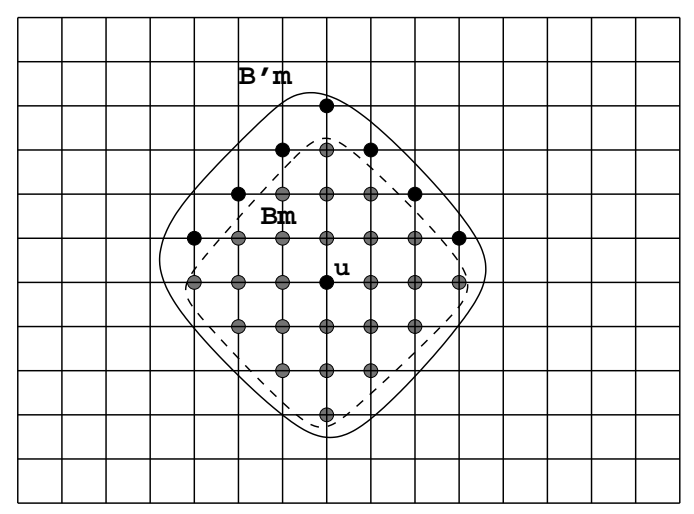

Figure 2: $B_{m}$ and $B_{m}^{\prime}$ in the case where $k=7$ (ie, $m=3$ )

The upper bound is obtained using the following coloring : for any vertex $u=(x, y)$ in $V\left(G_{2}\right)$, $u$ is assigned color $c(u)=x+k y \bmod p$, where $p=2 m^{2}+4 m+2$. We now prove by contradiction that any two distinct vertices $u_{1}$ and $u_{2}$ lying at distance less than or equal to $k$ in $G_{2}$ cannot be assigned the same color by the above coloring. Suppose, to the contrary, that $1 \leq d(u, v) \leq k$, where $u=\left(x_{1}, y_{1}\right)$ and $v=\left(x_{2}, y_{2}\right)=\left(x_{1} \pm s, y_{1} \pm t\right)$, with $s, t \geq 0$ such that $s+t=d(u, v)$. Let $\kappa=d\left(u_{1}, u_{2}\right)=s+t$ (hence $\left.1 \leq \kappa \leq k\right) . c\left(u_{1}\right)=c\left(u_{2}\right)$ is equivalent to $x_{1}+k y_{1} \equiv x_{1} \pm s+k\left(y_{1} \pm t\right)$ $\bmod p$, that is $\pm s \pm k t \equiv 0 \bmod p$. We distinguish two cases here, depending on the signs of the expression : 
Case $1: s+k t \equiv 0 \bmod p($ also equivalent to $-s-k t \equiv 0 \bmod p)$. Hence we have $\kappa+(k-1) t \equiv 0$ $\bmod p$. In other words, $\kappa+(k-1) t=\alpha p$. However, it can be easily seen that $\alpha>0$, since $\kappa \geq 1$, $k \geq 1$ and $t \geq 0$. Moreover, if $\alpha \geq 2$, then we would have $\kappa+(k-1) t \geq(k+1)^{2}$, which is impossible since $t \leq k$ and $\kappa \leq k$. Hence, the only possibility is $\alpha=1$, that is $\kappa+(k-1) t=\frac{(k+1)^{2}}{2}$. If $k=1$, this gives $\kappa=2$, which contradicts the fact that $\kappa \leq k$. Now if $k \geq 3$, we get the following equality : $t=\frac{k-1}{2}+2+\frac{2-\kappa}{k-1}$. However, $t$ must be an integer ; $k$ being odd, we just have to check that $\frac{2-\kappa}{k-1}$ is a (possibly negative) integer. It is easy to see that $-1<\frac{2-\kappa}{k-1}<1$, since $k \geq 3$. Thus, the only possibility is $\frac{2-\kappa}{k-1}=0$, that is $\kappa=2$. In that case, we end up with $t=\frac{k-1}{2}+2$, that is $t>2$ since $k \geq 3$. However, $\kappa=s+t$, with $s \geq 0$. Hence $\kappa>2$, a contradiction since we supposed $\kappa=2$.

Case $2: s-k t \equiv 0 \bmod p($ also equivalent to $-s+k t \equiv 0 \bmod p)$. Hence we have $\kappa-(k+1) t \equiv 0$ $\bmod p$, that is $t=\frac{\kappa}{k+1}+\alpha p, \alpha$ being a (possibly negative) integer. However, since $t \leq k$ and $p>k$, it follows that $\alpha<1$; and since $t \geq 0$, it follows that $\alpha>-1$. Thus $\alpha=0$; but in that case, $t=\frac{\kappa}{k+1}$, a contradiction since $t$ must be an integer and since $1 \leq \kappa \leq k$.

Thus, we conclude that, using the above coloring, no two vertices at distance less than or equal to $k$ in $G_{d}\left(n_{1}, \ldots, n_{d}\right)$ can be assigned the same color. In other words, the proposed coloring is a $k$-distance coloring for $G_{2}$. Since it uses $p=2 m^{2}+4 m+2=\frac{(k+1)^{2}}{2}$ colors, we conclude that $\chi_{k}\left(G_{2}\right) \leq \frac{(k+1)^{2}}{2}$. Altogether, we have that $\chi_{k}\left(G_{2}\right)=\frac{(k+1)^{2}}{2}$ and the theorem is proved.

\section{Conclusion}

In this paper, we have determined the value of $a\left(\mathcal{G}_{d}\right)$ for the family $\mathcal{G}_{d}$ of $d$-dimensional grids, $d \geq 1$; we have also determined the value of $\chi_{2}\left(\mathcal{G}_{d}\right), d \geq 1$ and $\chi_{k}\left(\mathcal{G}_{2}\right), k \geq 1$. The results on acyclic coloring are given by determining a universal lower for $a(G)$ on any graph $G$, that meets an upper bound given by an appropriate coloring. More precisely, the bounds meet for "sufficiently large" grids $G_{d}\left(n_{1}, \ldots, n_{d}\right)$, that is when $\sum_{i=1}^{d} \frac{1}{n_{i}} \leq 1$. If this is not the case, the bounds differ by an additive factor equal to $1-\left\lfloor\sum_{i=1}^{d} \frac{1}{n_{i}}\right\rfloor$. Adapted to $d$-dimensional hypercubes $H_{d}$, these bounds are a factor 2 away.

All the upper bound results (and also some results on star coloring mentioned in Remark 1) rely on an appropriate coloring that makes use of the representation of each vertex of $G_{d}\left(n_{1}, \ldots, n_{d}\right)$ by its coordinates in each dimension. This coloring method is adapted depending on the considered graph coloring, but remains the same in spirit. We suspect that it can be extended to other kinds of coloring, or to more general results concerning $k$-distance coloring in $d$-dimensional grids, for any $k$ and $d$.

We end this article by the following open problem : what is the exact value of $a\left(H_{d}\right)$ for hypercubes of dimension $d$ ? Table 1 suggests that $2+\left\lfloor\frac{d}{2}\right\rfloor$ is a good candidate ; we have not been able to prove it so far.

\section{References}

[AM00] N. Alon and B. Mohar. The chromatic number of graph powers. Combinatorics, Probability and Computing, 2000. To appear.

[BKW99] O.V. Borodin, A.V. Kostochka, and D.R. Woodall. Acyclic colourings of planar graphs with large girth. J. London Math. Soc., 60 (2):344-352, 1999.

[Bor79] O.V. Borodin. On acyclic colorings of planar graphs. Discrete Mathematics, 25:211-236, 1979.

[Bur79] M.I. Burstein. Every 4-valent graph has an acyclic 5 coloring (in russian). Soob̌̌c. Akad. Nauk Gruzin, SSR 93:21-24, 1979. 
[FRR02] G. Fertin, A. Raspaud, and B. Reed. Star coloring of graphs. LaBRI, Technical report, 2002. Submitted for publication.

[Grü73] B. Grünbaum. Acyclic colorings of planar graphs. Israel J. Math., 14(3):390-408, 1973.

[KDP00] D.S. Kim, D.-Z. Du, and P.M. Pardalos. A coloring problem on the $n$-cube. Discrete Applied Mathematics, 103:307-311, 2000.

[Sop97] E. Sopena. The chromatic number of oriented graphs. Mathematical Notes, 25:191-205, 1997. 